\title{
Intranasal dexmedetomidine vs intranasal midazolam for premedication in paediatric patients undergoing surgery under general anaesthesia
}

\author{
Josemine Davis ${ }^{1}$, Thaneshwar Rijal ${ }^{2}$, Amit Rai ${ }^{3, *}$ \\ ${ }^{\mathbf{1}}$ Associate Professor, Dept. of Anaesthesia, Command Hospital, Pune, Maharashtra, ${ }^{\mathbf{2}}$ Assistant Professor, Dept. of Anaesthesia, \\ Shree Birendra Hospital, Kathmandhu, Nepal, ${ }^{3}$ Associate Professor, Dept. of Anaesthesia, Neval Hospital, INHS Asvini Colaba, \\ Mumbai, Maharashtra, India
}

*Corresponding Author:

Email: drraiamit@gmail.com

Received: $20^{\text {th }}$ March, 2018

Accepted: $27^{\text {th }}$ March, 2018

\begin{abstract}
Introduction: This prospective, randomized, double-blind study was conducted with an aim to compare the efficacy of intranasal dexmedetomidine and intranasal midazolam as premedication in uncooperative children undergoing elective surgery under general anaesthesia.

Materials and Methods: One hundred and twenty children in American Society of Anesthesiology classification (ASA) physical status I \& II, in the age group of 2-12 years who were planned to undergo surgery under general anaesthesia, were included in the study and were randomly assigned to one of the two groups. Group D received $1 \mathrm{mcg} / \mathrm{kg}$ of IN dexmedetomidine while group M received $0.2 \mathrm{mcg} / \mathrm{kg}$ of IN midazolam for premedication. The patient's sedation status, separation anxiety and mask acceptance along with the hemodynamic parameters were noted.

Results: Satisfactory sedation was achieved in $56 \%$ children in group M and $65 \%$ children in group D ( $p>0.05$ ). Compared with the children in group $\mathrm{M}$, those in group D had significantly better satisfactory anxiolysis at the time of separation from the parents (56 vs 50; $\mathrm{p}<0.05$ ). Mask acceptance was better in group D ( 55 children in group D vs 36 children in group M; $<$ 0.05). Before parental separation, the heart rate was significantly lower in the dexmedetomidine group as compared to midazolam group $(102.88 \pm 6.07$ vs $110 \pm 7.34 ; \mathrm{p}<0.05)$.

Conclusion: Intranasal midazolam and dexmedetomidine are both effective as premedication in children undergoing surgery. However, intranasal dexmedetomidine offers better effect on sedation, alleviating separation anxiety and acceptance of facemask during induction than intranasal midazolam.
\end{abstract}

Keywords: Intranasal, Dexmedetomidine, Midazolam.

\section{Introduction}

Children react to their unfamiliarity with the new environment of operation theatre and become anxious being separated from the parents. One of the challenges for anesthesiologists is to minimize distress for children in the operating room environment and to facilitate a smooth induction of anesthesia. This is best accomplished by non-pharmacological means including developing a good rapport with the child and parents and making the environment kid friendly. However there is always a child who is difficult to control and pharmacological means may be very effective. This may be accomplished by prior administration of a sedative drug as premedication or parental presence during transfer to the operation theatre. ${ }^{1}$ Midazolam is the most commonly drug for premedication. Alpha-2 $(\alpha-2)$ agonists like clonidine and dexmedetomidine have also been widely used as premedication in pediatric anesthesia.

Midazolam, a short-acting benzodiazepine, provides effective sedation, anxiolysis, and varying degrees of anterograde amnesia; however, adverse effects such as postoperative behavioral changes, hiccups, paradoxical hyperactive reactions and respiratory depression have been observed in higher doses. ${ }^{2}$ Dexmedetomidine, a potent and highly selective $\alpha-2$ receptor agonist, provides anxiolytic, analgesic, sedative effects without respiratory depression. It also reduces stress responses to surgical and other medical procedures and decreases the incidence of agitation at emergence. ${ }^{3}$ Midazolam can be given by various routes including intravenous, oral and intranasal (IN). In our center we usually take intravenous access after inhalational induction. So the oral and nasal route is usually preferred for premedication drugs. IN drug delivery is easy, convenient and reduces first pass metabolism. IN administration of midazolam is easy, effective, quick, and noninvasive. IN dexmedetomidine is efficacious and well tolerated in healthy volunteers and is therefore a useful premedication in children.

The objective of this investigation was to compare the efficacy of two premedication regimens in uncooperative children posted for surgery under general anaesthesia. The children received either intranasal dexmedetomidine or intranasal midazolam.

The primary end point of this study was to measure and compare the sedation status and separation anxiety at separation from the parents. The secondary end point was to compare mask acceptance during induction in the operating room. 


\section{Materials and Methods}

The study was conducted in a tertiary care teaching hospital from August 2014 to July 2016.The institutional ethical review committee approval was obtained. An informed consent was obtained for each patient from either of the parents. Children in American Society of Anesthesiology classification (ASA) physical status I \& II, in the age group of 2-12 years who were planned to undergo surgery under general anaesthesia, were included in the study. The patients with known hypersensitivity to benzodiazepines or alpha 2 receptor agonist drugs, congenital diseases (congenital heart diseases, Down's syndrome etc.), child with developmental delay and/or with neurological disease (cerebral palsy, raised ICP etc.) were excluded in the study. 120 children were randomized using computer generated random number tables into two groups of 60 children. Group D received $1 \mathrm{mcg} / \mathrm{kg}$ of IN dexmedetomidine while group $\mathrm{M}$ received $0.2 \mathrm{mcg} / \mathrm{kg}$ of IN midazolam for premedication. The children were placed along with the parent in a full resuscitation facility during drug administration. The calculated amount of drug was diluted with $0.9 \%$ normal saline to make final volume of $1 \mathrm{ml}$. Half of the volume of the drug was administered in each nostril in the presence of his/her parent, using a needleless one $\mathrm{ml}$ syringe. An independent observer recorded the baseline $\mathrm{SpO}_{2}$ and pulse rate using a pulse oximeter. The child was shifted to the operating room after 30 minutes. A pulse oximeter was kept attached and the child was monitored. At the time of separation from the parents, the sedation of the child was assessed and noted using a four-point sedation score. A score of 3 and above was taken as the satisfactory sedation. The separation anxiety of the patient was also assessed during this time by using a four-point anxiety scale. A score of 2 or less was taken as satisfactory anxiolysis. On the operation table, acceptance of facemask at the time of induction was noted with three-point anxiety scale. Satisfactory mask acceptance during induction was mask acceptance score less than or equal to 2 . The different scores for assessment are mentioned in table 1. Standard anaesthetic technique was followed for all patients after shifting the patient to the operation theatre.

\section{Statistical Analysis}

Sample size was calculated based on a previous study (7). Accordingly a sample size of 60 patients per study group was estimated to achieve a power of $80 \%$ $($ alpha $=0.05)$. Statistical analysis was done with SPSS software version 20.0. Quantitative data like age, pulse rate, $\mathrm{SpO}_{2}$ was presented in the form of mean and standard deviation. Comparison between two study groups was done using an unpaired Student's t-test. Qualitative data was presented as frequency or percentage. Using the p-values obtained through student t-test did analysis or their significance was done. Two independent variables were compared using the student t-test. The Mann-Whitney U test was used for comparing the sedation score, anxiety score and degree of mask acceptance between two groups. A $p$ value $<0.05$ was considered statistically significant.

\section{Results}

Two groups were comparable with respect to age and body weight [Table 2]. The mean age in group D and group $M$ was $4.53 \pm 2.55$ years and $5.45 \pm 2.82$ years respectively. The mean body weight in group $\mathrm{D}$ was $15.28+5.82 \mathrm{~kg}$ and the mean age in group $\mathrm{M}$ was $17.02+4.78 \mathrm{~kg}$. Satisfactory sedation was achieved in 34 children (56\%) in group $\mathrm{M}$ and 39 children (65\%) in group D. However the difference was not statistically significant $(p>0.05)$ [Fig. 1]. However, compared with the children in group $M$, those in group $D$ had significantly better satisfactory anxiolysis at the time of separation from the parents ( 56 children vs 50 children; $\mathrm{p}<0.05)$. [Fig. 2]. Similarly there was a significant difference between two groups $(p<0.05)$ in terms of mask acceptance by the children. 55 children in group D vs 36 children in group $M$ ) had satisfactory mask acceptance. [Fig. 3] Before premedication the heart rate in both the groups was comparable (Group D $113.73 \pm$ 8.22 vs Group M $111.12 \pm 8.29$ beats/minute; $p>0.05$ ). However, before parental separation, the heart rate was significantly lower in the dexmedetomidine group as compared to midazolam group $(102.88 \pm 6.07$ vs $110 \pm$ 7.34; $p<0.05$ ). [Table 3, Fig. 4] There was no significant difference between mean $\mathrm{SpO}_{2}$ values in group $\mathrm{D}$ and group $\mathrm{M}$ before premedication $(\mathrm{p}=0.0682)$ and before induction $(\mathrm{p}=0.0785)$ respectively. The mean $\mathrm{SpO}_{2}$ showed a marginal fall from $98.88 \%$ to $98.37 \%$ after premedication in group D and from $98.82 \%$ to $98.32 \%$ after premedication in group $\mathrm{M}$. These changes in $\mathrm{SpO}_{2}$ were clinically insignificant. [Table 4]

\section{Table 1: Sedation, separation anxiety and mask acceptance scores}

\begin{tabular}{|l|l|}
\hline Four-point sedation score (1) & Four-point separation anxiety scale $(3)$ \\
\hline $1=$ Alert, awake & $1=$ Calm and cooperative \\
$2=$ Drowsy, sleepy, lethargic & $2=$ Anxious but could be reassured \\
$3=$ Asleep but responds to mild prodding or shaking & $3=$ Anxious and could not be reassured \\
$4=$ Asleep and does not respond to mild prodding or & $4=$ Crying or resisting \\
shaking & \\
\hline
\end{tabular}




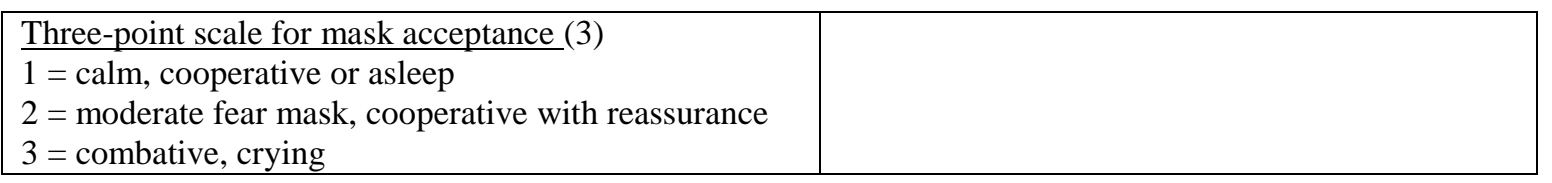

Table 2: Demographic parameters

\begin{tabular}{|l|c|c|c|}
\hline $\begin{array}{c}\text { Demographic } \\
\text { Parameter }\end{array}$ & $\begin{array}{c}\text { Dexmedetomidine group } \\
\text { (Group D) } \mathbf{N = 6 0}\end{array}$ & $\begin{array}{c}\text { Midazolam group (Group M) } \\
\mathbf{N = 6 0}\end{array}$ & P value \\
\hline Body weight $(\mathrm{Kg})$ & $15.28 \pm 5.82$ & $16.87 \pm 4.78$ & 0.106 \\
\hline Age (Yrs) & $4.53 \pm 2.55$ & $5.54 \pm 2.82$ & 0.064 \\
\hline
\end{tabular}

Table 3: Heart rate variation

\begin{tabular}{|l|c|c|c|c|c|}
\hline \multirow{2}{*}{ Heart rate } & \multicolumn{2}{|c|}{ Group D (n=60) } & \multicolumn{2}{c|}{ Group M (n=60) } & \multirow{2}{*}{$\begin{array}{c}\text { Inter group p- } \\
\text { value }\end{array}$} \\
\cline { 2 - 5 } & Mean & SD & Mean & SD & 0.085 \\
\hline Before premed & 113.73 & 8.22 & 111.12 & 8.29 & $<0.001$ \\
\hline Before separation & 102.88 & 6.07 & 110.75 & 7.34 & $<0.75$ \\
\hline
\end{tabular}

Table-4: $\mathrm{SpO}_{2}$ variation after premedication in two groups

\begin{tabular}{|l|c|c|c|c|c|}
\hline \multirow{2}{*}{ SpO$_{2}$} & \multicolumn{2}{|c|}{ Group D $(\mathbf{n}=60)$} & \multicolumn{2}{c|}{ Group M (n=60) } & \multirow{2}{*}{ Inter group p-value } \\
\cline { 2 - 5 } & Mean & SD & Mean & SD & \\
\hline Before premed & 98.88 & 0.88 & 98.82 & 0.89 & 0.682 \\
\hline Before induction & 98.37 & 1.01 & 98.32 & 1.00 & 0.785 \\
\hline
\end{tabular}

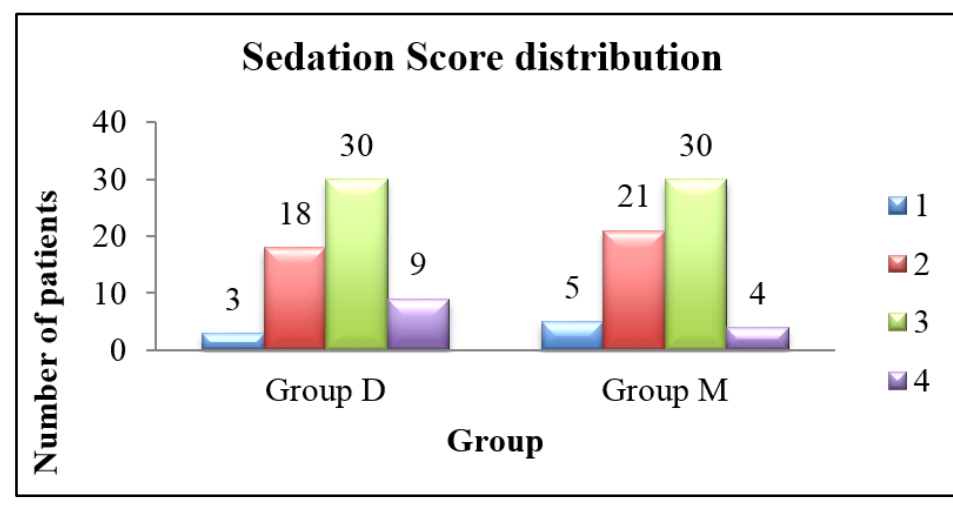

Fig. 1: Distribution of the sedation score in the two groups

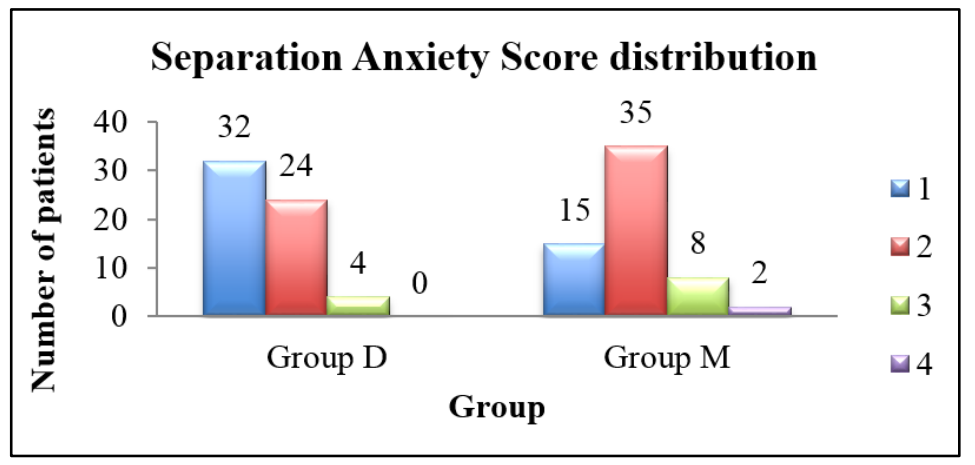

Fig. 2: Distribution of separation anxiety score in the two groups 


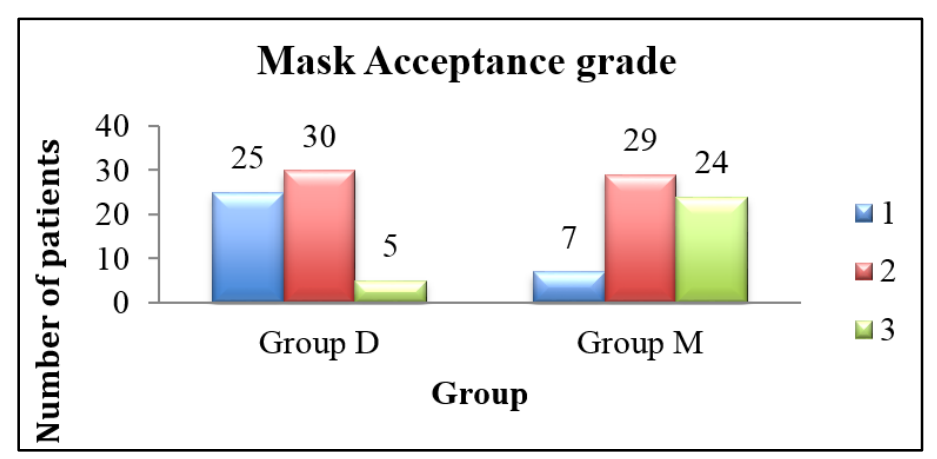

Fig. 3: Distribution of grade of mask acceptance

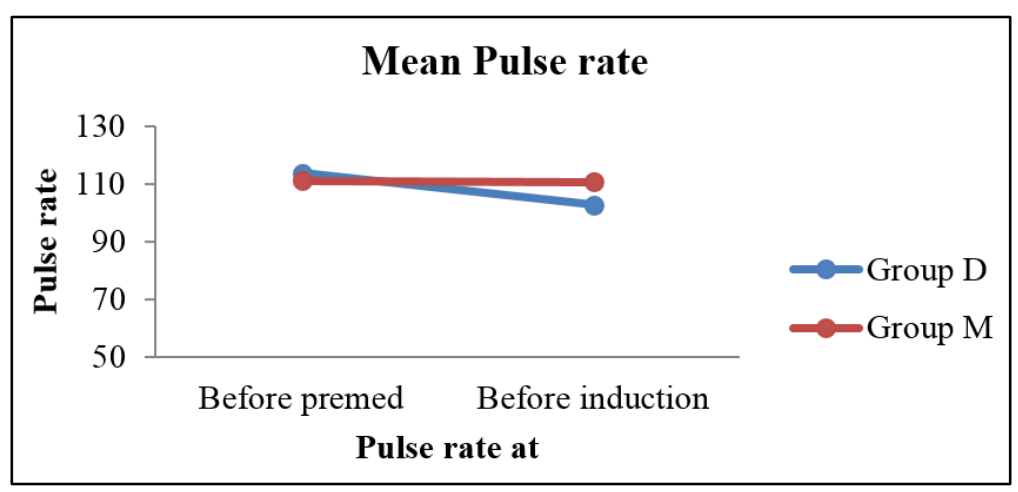

Fig. 4: Changes in the heart rate after premedication

\section{Discussion}

Dexmedetomidine and midazolam can be used as a premedication in children through various routes like intravenous, oral, intramuscular, intranasal, transmucosal etc. Intranasal route of administration is a relatively easy, painless and non-invasive route unlike the intravenous or intramuscular route; produces more rapid onset of action than the oral route and has higher bioavailability as compared to oral route. Children cooperate and get assured better as compared to intravenous or intramuscular route. So, we aimed to compare the effects of these two drugs administered through intranasal route to draw the inferences on their efficacy. The intranasal dose of dexmedetomidine $(1 \mathrm{mcg} / \mathrm{kg})$ was decided based on previous studies done like a study done by Yuen $\mathrm{M}$ et al, ${ }^{2}$ Talon et al ${ }^{4}$ while standard intranasal dose of midazolam $0.2 \mathrm{mg} / \mathrm{kg}$ was taken.

Our results showed that $65 \%$ of children achieved satisfactory sedation in the intranasal dexmedetomidine versus $57 \%$ children in the intranasal midazolam group, though not statistically significant. Probably if a higher sample size is taken, there may be a statistically significant change.

Schmidt et al in their study also ${ }^{5}$ did not find any difference in sedation between intranasal dexmedetomidine and oral midazolam while Ashraf M Ghali et al, ${ }^{6}$ Yuen $\mathrm{M}$ et al, ${ }^{2}$ found increased sedation in the intranasal dexmedetomidine group as compared to oral midazolam group. This difference could have resulted from the different scale used for assessment of sedation. We used a 4-point sedation scale similar to in the study of Schmidt et al while those studies used 6point sedation scale. The studies done by Deepak Singla et al., ${ }^{7}$ Shad et al, ${ }^{8}$ Hebaallah et al., ${ }^{9}$ Darsana D. Patil et $\mathrm{al}^{10}$ found that the sedation achieved in intranasal dexmedetomidine group was better than that in intranasal midazolam group. Similarly, Sundaram et $\mathrm{al}^{11}$ in their study found that intranasal dexmedetomidine achieved more satisfactory sedation than in intranasal midazolam group. Intranasal dexmedetomidine achieved satisfactory sedation during the time of parental separation but the children were distressed at the time of induction. The study found that the dose may not be adequate to achieve the adequate depth of sedation level and the child may get awaken during the transfer from the holding area to the induction table.

Regarding child-parent separation score, children in group D were more satisfactorily separated from parents than in group M. Our results are in consensus with Ashraf M Ghali et $\mathrm{al}^{6}$ and Akin et al, ${ }^{3}$ Darsana et al, ${ }^{10}$ Heballah et al,. ${ }^{9}$ Shad et al. ${ }^{8}$ found that the children in dexmedetomidine group were more sedated during parental separation than in intranasal midazolam group and separation from parents was easier in dexmedetomidine group as compared to midazolam group. Sundaram et $\mathrm{al}^{11}$ in their study found that in intranasal dexmedetomidine group the separation from the parents was more easier compared to intranasal midazolam group however the child became awake during the transfer of patient from pre holding area to 
the operation room. This might be due to the inadequacy of the dose of dexmedetomidine.

With respect to the acceptance of facemask during induction, our study found that there is statistically significant difference between the two groups. Intranasal dexmedetomidine provides a better effect on the acceptance of facemask than intranasal midazolam. This was congruent to the result with the study done by Hebaallah et $\mathrm{al}^{9}$ and Deepak Singla et $\mathrm{al}^{7}$ who also found that the mask acceptance was better after intranasal dexmedetomidine compared with midazolam 30 min after drug administration. Contrary to these study, Akin et al. ${ }^{3}$ in their a study comparing intranasal dexmedetomidine and midazolam in children, administered 45-60 min before the induction of anaesthesia found that midazolam produced superior mask induction than dexmedetomidine.

Dexmedetomidine decreases sympathetic outflow thereby decreasing the circulating catecholamine levels and increases cardiac vagal activity. So, it would be expected to cause a decrease in heart rate in patients receiving dexmedetomidine. Our study showed that intranasal dexmedetomidine causes a significant reduction in the heart rate as compared to intranasal midazolam. However the decrease in heart rate was clinically insignificant and there was no incidence of bradycardia. Deepak Singla et $\mathrm{al}^{7}$ in their study did not find any significant changes in the heart rate after premedication in both groups. Hebaallah et $\mathrm{al}^{9}$ and Sundaram et al $^{11}$ found significant bradycardia after premedication with dexmedetomidine while there was no significant change in heart rate in midazolam group.

Midazolam acts on GABA- mimetic system and decreases the respiratory drive in a dose-dependent manner. The dose of midazolam administered in our study was not significantly high to cause the decrease in respiratory drive. The arterial oxygen saturation was well maintained throughout the perioperative observation period. All the previous studies done to compare the intranasal dexmedetomidine and midazolam showed no significant changes in $\mathrm{SpO} 2$ after administration of the premedication. Darsana et $\mathrm{al}^{10}$ found that after intranasal midazolam there was fall in respiratory rate without any change in $\mathrm{SpO} 2$ level. Akin et $\mathrm{al}^{3}$ and Hebaallah et $\mathrm{al}^{9}$ did not find statistically significant decrease in the respiratory rate or $\mathrm{SpO} 2$ below $95 \%$ in both the groups.

\section{Limitations of the Study}

The major limitation of this study was the timing of the drug administration, as dexmedetomidine was not allowed to reach its peak effect before mask induction. For dexmedetomidine, this length of time may be too short, and for some children, the drug may be yet to have an effect. It might have possible to note greater sedative effects in the intranasal dexmedetomidine group if we had waited longer. But if we would have waited longer, than the effect of midazolam would have disappeared. The other limitation of this study is the use of invalidated three- or four-point scales. When using these scales, we encountered some difficulties in the evaluation of children. For example, if the child was crying but not combative, we found it hard to decide what rating to give on the mask induction scale. It may be necessary to use more valid scales. In our study, both midazolam and dexmedetomidine were administered in the form of intranasal drops. The use of spray/metered dose atomizer would give better absorption and greater bioavailability of intranasal drug.

\section{Conclusion}

In our study, we found that the midazolam and dexmedetomidine are both effective as premedication in paediatric age groups planned for general anesthesia. However, intranasal dexmedetomidine offers better effect on sedation, alleviating separation anxiety and acceptance of facemask during induction than intranasal midazolam.

\section{References}

1. V. M. Yuen TWH, M. G. Irwin, T.J. Yao, G. L. Wong and M. K. Yuen. Optimal timing for the administration of intranasal Dexmedetomidine for premedication in children. Anaesthesia. 2010;(65):922-9.

2. Yuen VM, Hui TW, Irwin MG, Yuen MK. A comparison of intranasal dexmedetomidine and oral midazolam for premedication in pediatric anesthesia:a double-blinded randomized controlled trial. Anesthesia \& analgesia. 2008;106(6):1715-21.

3. Akin A, Bayram A, Esmaoglu A, Tosun Z, Aksu R, Altuntas R, et al. Dexmedetomidine vs midazolam for premedication of pediatric patients undergoing anesthesia. Paediatric anaesthesia. 2012;22(9):871-6.

4. Talon MD, Woodson LC, Sherwood ER, Aarsland A, McRae L, Benham T. Intranasal dexmedetomidine premedication is comparable with midazolam in burn children undergoing reconstructive surgery. Journal of burn care \& research: official publication of the American Burn Association. 2009;30(4):599-605.

5. Schmidt AP, Valinetti EA, Bandeira D, Bertacchi MF, Simoes CM, Auler JO, Jr. Effects of preanesthetic administration of midazolam, clonidine, or dexmedetomidine on postoperative pain and anxiety in children. Paediatric anaesthesia. 2007;17(7):667-74.

6. Ghali AM, Mahfouz AK, Al-Bahrani M. Preanesthetic medication in children: A comparison of intranasal dexmedetomidine versus oral midazolam. Saudi Journal of Anaesthesia. 2011;5(4):387-91.

7. Singla D, Chaudhary G, Dureja J, Mangla M. Comparison of dexmedetomidine versus midazolam for intranasal premedication in children posted for elective surgery: a double-blind, randomised study. Southern African Journal of Anaesthesia and Analgesia. 2015;21(6):154-7.

8. Sheta SA, Al-Sarheed MA, Abdelhalim AA. Intranasal dexmedetomidine vs midazolam for premedication in children undergoing complete dental rehabilitation: a double-blinded randomized controlled trial. Paediatric anaesthesia. 2014;24(2):181-9.

9. Abdelmoneim H, Hamouda S, Mahfouz G, Hashem A. Intranasal dexmedetomidine versus midazolam in preoperative sedation for noncomplex pediatric 
congenital cardiac surgeries. Research and Opinion in Anesthesia and Intensive Care. 2016;3(3):129-37.

10. Darshna D. Patel L, M.R.Upadhyay. Pre anaesthetic medication in children A comparison of intranasal dexmedetomidine versus intranasal midazolam. The Journal of Medical Reseach. 2015;1(2):59-63.

11. AL Meenakshi Sundaram VMM. A Comparative Evaluation of Intranasal Dexmedetomidine and Intranasal Midazolam for Premedication in Children : A Double Blind Randomised Controlled Trial. Journal of Indian Dental Association. 2011;5(7):777-81.

\begin{tabular}{l}
\hline How to cite this article: Davis J, Rijal T, Rai A. \\
Intranasal dexmedetomidine vs intranasal \\
midazolam for premedication in paediatric \\
patients undergoing surgery under general \\
anaesthesia. Indian J Clin Anaesth. \\
2018;5(3):315-320. \\
\hline
\end{tabular}

